\title{
Myeloid Neoplasms with Germline Predisposition
}

National Cancer Institute

\section{Source}

National Cancer Institute. Myeloid Neoplasms with Germline Predisposition. NCI

Thesaurus. Code C130038.

A term that refers to myelodysplastic syndromes, myelodysplastic/myeloproliferative neoplasms, and acute myeloid leukemias that are associated with germline mutations and are familial. 